\title{
Namenregister
}

\begin{tabular}{|c|c|}
\hline 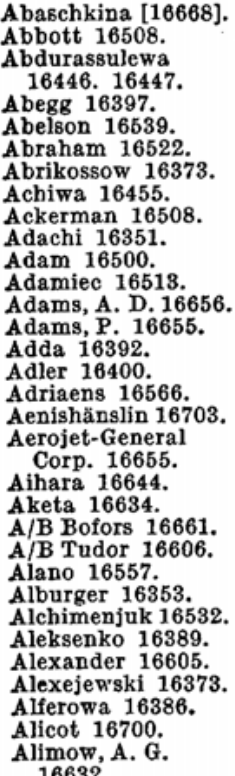 & 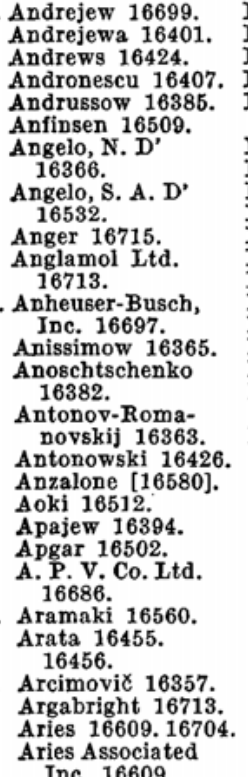 \\
\hline
\end{tabular}

$16632 . \quad$ Inc. 16609

Alimow, J. I. 16373. Armour \& Co.

Allanson 16569 .

Allen, C. F. H. 16473.

Corp. 16654 . 16659 .

Alpert, E. 16579.

Alpert, N. 16712 .

Alväger 16357 .

Ambegaokar 16542. Atlantic Refining

Amberg 16388.

American Cyanamid Atlas Powder Co. Co. 16579. 16580. 16696 . 16627. 16655. Austen 16528.

American Marine Avedikian 16611.

Paint Co. 16668. Awe 16565 .

American Oil Co. 16652.

American Viscose Corp. 16704.

Amiel 16445 .

Amirthalingam 16390.

Amlie 16380.

Amme 16419 .

Ammon 16545.

Anantakrishnan 16692.

Awtuchowa 16587.

Babakow 16635 .

Babarykin 16632 .

Babkine 16376.

Bacher 16647.

Bacon 16433.

Bádescu 16597.

Badische Anilin.

\& Soda-Fabrik

Akt.-Ges. 1660

16606. 16658.

16661. 16662 .

16667. 16672 .

16705 .

Andersen 16530.

Anderson, A. E. 16604.

Anderson, A. S.

16713.

Anderson, A. W.

16676.

Anderson, J. L. 16671.

Andersson 16699.

Andlaw 16529 .

Andrade, E. G. de 16613.

Badman 16526.

Baenitz 16570.

Baerlecken 16646 .

Bagdon 16555 .

Bagnetto jun. 16651.

Bagrjanski 16642.

Bahadri 16541.

Bahrs 16555.

Bai 16527.

Bailar jun. 16403. Bailey 16666 .

Andrade,E. N. Da C. Bailie 16609.

Baily 16662.
Bair 16853.

Baiulescu 16587.

Baker H. Begman 16631.

Baker Castor Oil Co. Behringer 16392. 16661.

16587.

Balaguer 16605 .

Balandina 16699 .

Balbi 16665 .

Ball 16509.

Ballhausen 16387 .

Ballou 16676.

Bally 16535 .

Balmer 16617.

Balogh 16486.

Balter 16633.

Banerjee, A. K. 16406.

Banerjee, N. G. 16707.

Bangham 16538.

Banks 16511

Banno 16407.

Banno 16407. 16662 . Bennion 16689.

Baranger 16352 .

Baranowski, A. 16621.

Baranowski, B. 16382.

Barber 16522.

Barbicane 16639 .

Bardin 16629

Bardyschew 16666.

Barger 16360.

Barker 16552.

Barnhart 16540 .

Barnoff 16618 .

Baron 16401.

Barré 16557.

Barros 16353.

Bartkus 16438 .

Bartoš 16581.

Bartsch 16694.

Barylo 16640.

Basanow 16631.

Beindorff 16673 .

16674.

Bejrablaya 16558.

Bel 16629.

Belcher 16581.

Belikow 16585 .

Beller 16540 .

Bellon 16390.

Bellwinkel 16614.

Below 16386.

Belz 16639.

Benade 16664.

Bender 16512.

Bendik 16565.

Benedict 16715 .

Beneventano

16350.

Béraud 16532.

Berengard 16587.

Beresin 16426.

Bergeon 16382.

Bergeret 16524.

Bergquist 16688 .

Bergsma 16489.

Berkowitsch

16615.

Berlin 16442 .

Bernard, M. L. J. 16703.

16386. Bolfa 16376.

Bolley 16661.

Bernstein, S, 16484. Bolstad 16676.

16486. 16580. Bonera 16377.

Bernstein, W. N. 16585.

Bertaccini 16536.

Besserre 16430.

Bestian 16676 .

Baschirow 16372. Bettini 16553.

Baschkirow 16377. Betz 16677.

Baschmakow 16633. Bhaskaran 16607 .

Basẽ 16585 .

Basic, Jne. 16618.

Bastien 16641.

Bhattacharya

Basu, D. P. 16701.

16534 16406.

Basu N. K 16407. Biddulph 16525 .

Batalowa 16669. Bidwell 16540 .

Batowa 16586 . Biellmann 16362

Bauer, H. H. 16584. Bienlein 16357 .

Bauer, L. 16432. Bieth 16528.

Bauer, S. 16565. Biggs 16540.

Baum 16532. Bighi 16591.

Baumeister 16362. Bigley 16553 .

Baumgarten 16648. Biller 16563.

Baun 16584.

Baur 16391.

Bawn 16490.

Bayfield 16688 .

Binder 16359.

Binkley 16502.

Binning 16652.

Birchall 16429.

Bayknt, F. 16591.

Baykut, S. 16591

Beale 16605 .

Beati 16667 .

Bechter 16405 .

Becker, K. A. 16384.

Bircher 16555 .

Bírecki 16621.

Birké 16400

Birkinshaw 1652

Birkofer 16524.

Birss 16375 .

Birtley 16583

Becker, W. H.16533. Bisagni 16456.

Beckett 16521.

Beckloff 16552 .

Bedreag 16349.

Bissliss 16544

Blackburn 16550 .

Blackwell 16662 .

Beecham 16694

Blade 16638 .

Beecham Research

Blair-West 16531. 16660.

Blane 16438.

enbecler

16351.

6588

16550

16615 .

Blood 16646.

16429.

Blumer 16548 .

Bobalek 16666.

B 16714.

Bodenheimer

16617.

Bodmer 16598.

Böhme 16458.

\& Soehne 16580 . 16628 .

Böttcher 16697. 16563.

Boose 16552.

Booth, B. A

16545. 16629

Borlera 16708.

Borzani 16519. 16649 .

Boudes 16431.

Boulin 16588.

Boyd 16528 .

Bozler 16546

Bozorth 16375 . 16376.

Bradley 16398.

Brady 16514.

Brauer 16397.

Brebec 16392.

Brecht 16547. 16611. 16541.

Blake, E. B. 16697. Breuer 16517 .
Bhattacharyya

, A. E. 16693.

Akt.-Ges, 16648. British United Shoe

oehringer, C. F.,

Boehringer, C. $\mathbf{H}$.

Boekelheide 16451

Bogdanow, 0 . W.

Bogdanow, W. P.

Booth, F. 16606

Boots Pure Drug Co. Ltd. 16578.

Bosch, R., G.m.b.

Boyer, P. D. 1668

Boyer, R. 16404.

Brammer 16394

Brasefield 16364.

Braun, H. A. 16564. Buob 16598.

Braun, M. P. 16640. Burch 16537.

Braun, T. 16585 . Burckhalter 16579.

raunschweigische

16684 .

Brennan 16713.

Brenner 16374.

Bretschneider, $\mathbf{G}$.

Bretschneider, $\mathbf{R}$.

ver 16419.

Brini-Frit\% 16432 .

Bristol I abora-

.

British Drug Houses 
Burton, C, L. 16624

Burton, L. K 16358.

Burton-Banning 16617.

Busch, G. 16370 .

Busch, Gissla 16693.

Busson 16524.

Butler 16603.

Butt, J. M. 16615

Butt, M. W. 16696

Buzas, A. 16178 .

Buzás, I. 16586.

Bychowskaja [16595].

Bykow 16636.

Bykowa 16587.

Cabot, G. L., Inc. 16606.

Cabrini 16512 .

Cahn 16557.

Cahoon 16606.

Cain 16520 .

California Research Corp. 16651. 16711.

Callomon 16412.

Calvert 16419 .

Cambridge 16585.

Camisa 16708.

Campbell 16561.

Canfield 16704.

Capp 16643.

Capps 16350.

Carbiener 16524.

Carborundum Co. 16620 .

Cardarelli 16544.

Carell 16402.

Carlson 16547.

Carlstedt 16626 .

- Carmerino 16579.

Carr 16712.

Carrà 16410.

Carranza jun. 16512.

Cartwright 16508

Castro Changa 16686.

Catch 16525

Cattaneo 16542 .

Causley 16646 .

Cavalieri 16505.

Cavallito 16576.

C. D. Patents Ltd. 16609.

Ceauşescu 16608.

Cech 16639.

Celanese Corp. of America 16661 . 16704.

Centre National de

la Recherche Scientifique 16659 .

Chakraborty 16106.

Chakraviarti 16523

Chalisowa [16595].

Chamberlain 1635

Chance, B. 16501 16506 .

Chance II, F. M. 16506

Chance, L. H 16701 .

Charles, J. A. 1664

Charles, M. 16514.

Chatterjee 16706.

Cheiker 16615 .

Chemical Construction Corp. 16611 .

Chemie Grünenthal G.m.b.H. 16579 .

Chemisches Werk Lowi G.m.b.H. 16662 .
Chemische Werke Albert 16876 . 16677. 16680.

Chemische Werke fuals A kt.-Ges. $16661,16673$.

Chemische Werke Witten G.m.b.H. 16570. 16703 .

Chem3trand Corp.

16673. 16674. 16705 .

Chen 16563.

Chiarotti 16362.

Chilton 16584.

Chilwell 16694.

Chinoin Gybzyszer es Vegyészeti Termúkek Gyára

R. T. Dr. Keresz- Conchie ty ty es Dr. Wolf

Chiodo 16377.

Chishnitschenko 16635.

Chitrik 16637.

Chitrow 16586.

Chmela 16613.

Chochlow 16631.

Chotkewitsch 16392.

Choudhury 16701.

Chovin 16572 .

Chow 16410.

howanskaja

16625.

Christenson 16549.

Christmann 16541.

Christochowitz 16631.

Chemical Corp. 16667. 16700 .

Commissariat à

l'Énerzie Atomique 16610

Commonwealth Enzineering Co. 16648. 16654.

Commonwealth, Industrial Gases Ltd. 16644.

Comp de Produits Chimiques

omp. Francaise Davies, D. G. 16713.

Dalgarno 16360 .

Dallinga 16425 .

16357.

Dance 16587.

Dandl 16604.

Dannley 16590

Darker 16569.

Das 16494.

Dasgupta, B. 16523. Dostrovsky 16354

Das Gupta, N. N. Doughty 16604.

Doumet 16620 .

Dowben 16545 .

Dow Chemical Co. 16657. 16659. 16673. 16705.

Davies, G. 16598.

Davis, D. D. 16376. Davis,

Davison 16360 .

Dawber 16416.

Dawson, D. J 16693 .

Conklin 1651:

Connell 16537.

Conochie 16692.

Consortium für Dawson, E. H.

Elektrochemische $\quad 16689$.

Industrie G.m.b. Dawson, J. B. H. 16661 .

16408.

Dawson, J. K. 16643.

Methane Corp. 16601.

16657 .

Cook 16716.

Coombes 16497.

Corbin 16695.

Corish 16678.

Chruściel, M. 16560. Cormier 16540 .

Chrusciel, T. 16560. Corneanu 16561.

Chun 16352 .

Ciba Akt.-Ges.

16575. 16579 . 16580,16680 .

Ciba Ltd. 16579. 16580. 16656. 16661. 16663 .

Ciba Soc. An. 16827. 16662

Cilag Akt.-Ges. 16578 .

Cilag-Chemie Akt.Ges. 16578 .

Cirillo 16697.

Cities Service Research \& Development Co. 16710 .

Clamroth 16677.

Clare 16638 .

Cornforth 16591 .

Corn Products Refining $\mathrm{Co}$.

Costiner 16535. Desnuelle 16514.

Dawydow 16361

Degkwitz 16557 .

Degtjarew 16374.

Deindoerfer 16884.

Delimarski 16381.

Delmon 16397

Demeester 16707.

Dementi 16359.

Demoen 16558.

Demott 16691.

Dennen 16545 .

Denton 16531.

16545.

Derieux 16519.

Coulombeau 16588. Desy 16655.

Coulson, C. A. 16410.

Coulson, R, A. 16534 .

Council of Scien tific \& Industrial

Research 16611.

Courtaulds Ltd. 16704.

Cousin 16676 .

Covington 16393.

Crafts 16623.

Cragle 16691.

Craig 16562. 16563.

, A. 16645. Crane 16509.

Creangá 16709.

Clark, H. E. 16541. Crewe 16389.

Clark, R. E. 16584. Crittall Mfg. Co.

Ltd. 16648 .

Csányi 16581.

Cubicciotti 16387.

16561.

Cullumbine 16559

Contes, M. E. 16543 . Curnow 16535.

Coates, R. V. 16389. Curragh 16428.

Cockburn 16608. Currier 16623.

Coffield 16611.

Coffman 16429 .

Coghlan 16531.

Curry 16676.

Cusack 16400.

Czuchajowski

, H. 16377, 16706 .

Cohen, S. I. 16627. Dacquisto 16550.

Cole 16548 .

Coleman 16496.

Colgate-Palmolive

Co, 16682.

DiBler 16523.

Dahlberg 16695.

Dahle 166555 .

Daidone 16661.

Colhoun 16551.

Daimler-Benz

Collier $16536 . \quad$ Ges. 16848.

Colloides Industriels Dairy 16696.

Françis 16682. Dalcq .16511.

Detrex Chemical

Industries, Inc.

16651.

Deutsche Gold- und Silber-Scheide. anstalt vormals Roessler 16611. 16657. 16661. 16662. 16668 . 16714.

Devi 16538.

Dewey 16513.1

Dezissert 16703.

Dhawan 16548.

Diamond 16355 .

Dickerman 16502. 
Farmer 16357. Farnworth 16701. Farrow 16502. Fatuewa 16378.

Faure 16596 .

Faurote 16654.

Favini 16410.

Fedossejewa 16491.

Feichting

Feinberg 16528.

Fejes 16596 .

Fekete 16629.

Feldgandler 16635 .

Feldman 16603.

Feliu 16638.

Felkin 16447.

Feneberg 16373.

Fernandes 16519 .

Fernelius 16384.

Ferrarro 16396.

Ferrero 16354.

Ferrer Pi 16590.

Ferrini 16514.

Ferroni 16354.

Feuer 16669.

Feyel-Cabanes 16558 .

Fick 16376

Fielding 16427.

Fields 16355 .

Finamore 16505 .

Finikow 16398.

Fink 16695.

Finley 16451.

Finocchiare 16350.

Finston 16505 .

Finzi 16350.

Firestone Tire \& Rubber Co. 16679. 16680.

Fa. P. Lechler 16715 .

Fischer, E. 0. 16402 .

Fischer, F. 16573.

Fischer, L. J. [16602].

Fitzer 16649 .

Fjellström 16420.

Flataker 16563.

Flavin 16511.

Flemings 16638 .

Fletcher 16419 .

Flexa Industria Materie Plastiche S.p.A. 16606 .

Fodor 16476 .

Fokt 16371.

Food Machinery \& Chemical Corp. 16675 .

Foote 16350 .

Foran 16616 .

Forchielli 16529.

Forker 16611.

Forman 16679.

Forsyth 16353 .

Gejew 16637

Gelbrecht 16565 .

Foster, G. I. 16675 .

Fothergill 16529.

Fowler 16549.

Fox 16513 .

Frackowiak 16414.

Fraenkel 16354

Frame 16396.

Frank, D. 16665.

Frank, M. 16583.

Frankel 16497.

Frant 16627

Franz, P. 16595.

Franz, R.-D. 165

Franzen 16420 . 16502.

Fratta 16708.

Frautschi 16351.

Freeman 16645 .

ridkin 16367.

Fried 16355.

16538.

16400 .

Froesch 16535.

rost 16580 .

rowein 16557 .

Frush 16420.

Fuchs 16662.

Fujita, I. 16362 . 16554

Fukuda 16353.

Fullwood 16629.

Fults 16623 .

Funk 16667.

Fuoss 16496.

Furiya 16515.

urman, D. M.

16631.

urman, G. J. 16508 .

Furukawa 16678

Furuya 16553.

16554

Tabbe 16503.

Gärtner 16649.

Gaidukow 16373.

Gaines jun. 16620

ajewa 16461.

Galan 16641.

Galatry 16363 .

Galatzeanu 1658

Corp. 16627.

Galy 16646 .

Gamboni 16482 .

Gamula 16688.

Gancevici 16522 .

Ganguly 16516 .

Garber 16388.

Garmaise 16434

Gasparix 16592.

Gastol 16621.

Gaudry 16434.

Gaydon 16361.

Geddes 16688 .

Geigy, J. R., 16626. 16662.

Geigy, J. R., Soc.

An. 16573.

Geiler 16629. 16654. 16620 . 16602 .

Genin 16359

George 16444

Geppert 16592.

Gerassimenko

16377.

Gerjuoy 16351.

Gernstein 16665.

Gerola 16528.
Freeport Sulphur Gerow 16444. Co. 16646. Gersdorff 16624 Frehse 16695 . Gertner 16497.

. 16507. Gesellschaft für

French, D. 16524. Iinde's Eis-

French, G. B. 16452. maschinen Akt.- Gourlay 16524

Freudenstein 16565. Ges. 16600. 16601. Goutier-Pirotte Gesellschaft für 16510.

Werkstoff- Goutte 16386.

Formung E. J.

Ritter K. G.

16677 .

friedman 16708. Geske 16414

Getz 16527.

Ghiorso 16355 .

Ghose 16407.

Giardini-Guidoni 16366.

Gies 16664 .

Giesecke 16592.

Gilbert, C. 16530 .

Gilbert, J. A. S.

$$
16507 .
$$

Gilde 16596.

Gildenskiold 16603.

Gillis 16594 .

Gillman 16530.

Grabar 16685 .

Gräвbeck 16543 .

Graf 16667 .

Gragson 16713.

Grainger 16690.

Granowski 16367.

Gratschewa, J. M. 16684 .

Gratschewa, 0.J. 16615 .

Gray 16576.

Grecu 16569.

Greelen 16597.

Greenburg 16606.

Greenstein 16569.

Greenwood 16393.

Gregor 16641.

Ginsburg [16595]. Grim 16618.

Ginzberg 16643. Grinberg 16633.

Gir 16450.

Giraud 16625 .

Girelli 16709 .

Girre 16540 .

Gittus 16630 .

Giuliani 16362.

Giulotto 16377 .

Gladkow 16630 .

Gladun 16369 .

Glässer 16514.

Gleason 16714.

Glenner 16516.

Glikin [16649]

(6)

Glucklich 16618 . 16405 .

Goding 16531.

öbel, J. 16700.

Goebel, K. 16409

Goetze, G. 16604

Goetze, Gerhard

16355.

Gokhole 16611.

Golant 16365 .

Gold, M. H. 16655 .

Gold, V. 16426.

Goldberg, E. D. 16410.

Goldberg, M. W. 16579 .

Grinnan 16569.

Grisolia 16511.

Grjasnowa 16427 .

Grochmalicka 16543 .

Groene 16675.

Gruber 16679.

Gründig 16541 .

Gruhle 16359 .

Grum 16583 .

Grundon 16481.

Gubert 16641.

Gudowitsch 1667

Gudynski 16556.

Guérin-Dumartrait

$$
16525 .
$$

Güthner 16357.

Guex 16681.

Guhl 16535 .

Guidoni 16514.

Guilbault 16586 .

Guillaud 16386 .

Guillaume 16519 .

Guillet 16625 .

Guillien 16594 .

Gujral 16548.

Guljajew 16639 .

Guller 16710

Gullman 16390.

Gundlach 16405 .

Gunn 16709.

Gurjew 16633 .

Guro 16368.

Gussew 16698.

oldsmith 16569.

Gussewa 16464 .

Goldstein, L. 16551. GuBstahlwerk

Goldstein, S. W.

Witten Akt.-Ges. 16601.

Goleblewski 16410, Gustavson 16400.

. Golubewa, A. D. 16683 .

General Aniline \& Golubewa, L. A. Film Corp. 16644. . 16366 .

Gyimesi 16357 .

eneral Electric Gontscharow, K. S. Gysin 16626. Co. 16605. 16619.

General MotorsCorp.

Gontscharow, P. G. Habib 16421.

16637.

Gontscharowa 16395.

Goodison 16630.

Goodman 16443.

16709. 16879. 16704.

Goodwin 16350 .

Gutowsky 16416 . 
Horowicz 16546. Horsley 16657. Horatmann 16391. Horton 16448 . Hoste 16594.

Hotelling 16438.

Houilleres du Bassi du Nord et du Pas-de-Calais 18676 .

Howatson 16365. Howitt 16702 . Goyman 16624 . Hsis 16545 . Hslao 16494.

Hubert 16408 . Hubmzyer 16538 .

Hüokel 16566. Hüttel 16104. 16105. Iwanowa 16665 . Huffman 16705 . Hugzins 16355 . rughes, E. C. 16715 .

Hughe3, F. A., \& Co. Ltd. 16863 .

Hughe3, N. A. 16177 .

Huzhes, W. B. 16710 .

Hull 16578 .

Halliger 16533.

Hummolstedt 16418.

Humphrey 1663 t. Humphriss 16692 . Hunsberger 16116 . Husain 16519 . Hutchinson 165 Tuting Reinoso 16686.

Hydorn 16477 . Ichige 16454. Ichihara 16515. Ieso, di 16539. Igantius 16543 . Ikeda 16520 Ikegami, H. 16365 . Jenning 1659. Ikegami, Y. 16565. Jerchel 16169.

Ikeno 16640 .

Illissawski 16371.

Ilver 16570 .

Imperial Chemical Industries Ltd. 16578. 16579 . 16650. 16857. 16660. 16862 . 16675. 16680 . 16687, 16716.

Imperial Chem Industries of 16654 .

New Zealand Ltd. Joly 16359 . 16849.

Indian Oxygen \& Acetylene Co. Private Ltd. 16809. Izákováa 16585.

Izumi 16579.

Jackson 16691.

Jacob 16574.

Järnefeldt 16513.

Jaffe 16353.

Jaffee 16638.

Jaffey 16358.

Jageneau 16558.

Jakimowa 16587 .

Jakob, F. 16801 .

Jakob, H.-G. 1670

James 16463.

Jankowska 16378. Kardaschew 16360. Kisgjak 16414.

Janowa 16130 .

Janzen 16610.

Janssen 16558

Jaroszewicz 1651

Jaquith 16696.

Jasper 16668.

Jaudon 16588.

Jawoisski 16634.

Jegorowa [16595].

Jennings 1669

Jermolenko 16698.

Jerofejew 16356.

Jeroschkina 16678 .

Jewell 16697.

Johannson 16357 .

Johnson, J. A. 16688.

Johnson, K. D. B.

Jonas, J. 16570.

Jonas, R. E. E. 16890

Jopson 16358.

Jorpes 16580.

Intermetall Gesell- Joseph, N. M. schaft fur Metall- 16574. urgie und Elektro- Josien 16412. nik m. b. H. Jouan 16540 . $16804 . \quad J u c k e r 16576$.

International Mine-Jude 16486 . rals \& Chomical Jumar 16469. Corp. 16681.

Internationella Siporex A/B 16619.

Invents Akt.-Ges. für Forschung und Patentrerwartung 16852

Ioffe, A. I. 16678 .

Ioffe, B. S. 16395

Ioffe, W. A. 16367. Jussfin 16631.

Ionescu-Mihşieşti Just 16134. 16522 .
.

unker, 0., G. m. b. H. 16614. [16489].

Jurjew, W. A. [16548].

Jurjew, W. J. Just 16134
Justi 16371
Kann 18869.

Keii 16380 .

Kellogg, D. A.

Keunen 16706 .

Khogali 16544.
Kabata 16620 . Kägi 16547.

Känzig 16377.

Kästner 16630 .

Kaiser 16524.

Kimura, Y. 16442.

16518.

Kondorski 16376

Kakushadze 16389. King, Edward J. Konowalow 16386.

Kalb 16842.

Kall-Chemie Akt.- King, H. K. 16688.

King, J. G, T. 16697 .

Kaliko 16707 .

Kaljanow 16642.

King, L. C. 16471 .

Kallistratos 16587. King, R. J. B.

Kálmán 16708.

16516.

King, T,

Kameda, Y. 16112. Kinneman jun.

Kamsnskaja 16677. 16715.

Kinsky 16510.

Kaminski 16517 .

Kanawetz 16669 .

Kanda 16561.

Kano 16354.

Kaneyuki 16565

Kanter 16364 .

Kapelewitseh

16587

16377.

Kipke 16592 .

Kiprianow 16167.

Kirenski 16374

Kirianenko 16392 . Koshemjakin 16587.

Kiritschenko 16699. Koshewnikow

Kirmaier 16668.

Kirrmann 16550

1641

Kiss 16356 . Kisselew, A. A.
16834.

Karapetjanz 16418. Kisselew, W. S.

Kargin 16491.

Karlson 16537 .

Karmin 16677.

Kitanishi 16494

Kitko 16590.

Karnauch 16597. Kiyooka 16649.

Kaschtschuk 16636. 16650.

Kaspras 16647. Kleber 16388.

Katayama 16561. Kleiber 16692.

Katchalski 16497. Klein 16615.

Katz, L. N. 16528. Kleinman 16368.

Katz, P. S. 16822. Klepping 16560.

Kaufman $16708 . \quad$ Kleschick 16548 .

Kavan 16585.

Kavanagh 16569 .

16386.

Klingenberg 165

Kljujew 1641

Kawersnewa 16498. Klotz 16420.

Kay, R. W. 16434. Klumpp 16626.

Kaye 16569 .

Kmetec 16510 .

Kazinczy, de 16631. Knapp 16542.

Keblusek 16604

Keefer 16424.

Keeler 16519.

Kehren 16703

Keil 16709.

Keiss 16635 .

Keller 16579.

Kellermann 16648. 16516.

Kellogg, M. W.

16605. 16649 .

16670. 16676

Kelly 16688.

Kemali 16555

Kemp 16690.

Kemsley 16392.

Kenney 16350 .

Keogh 16692 .

16684.

Kessler, G. 16363.

Kessler, K. G. 16360.

Kes3ler, M. F. 16708

Khairallah 16536. 16558.

18477.

Kikuchi 16560 .

tschenko

Kottasz 16686 .

Kowald 16668.

Kragh Nielsen 16567.

Krantz 16619

Krassowskaja 16391. 16392 .

Kratz 16800

Kratzer 16404

Kraus 16581.

Kolkunow 16350. Kure 16885.

Kurita 16600.

Kuroda 16414

Kurylowicz [16523].

Kurzer 16465.

Kuschnir 16397.

usmin 16444.

16450.

Kusmowitsch 16381.

Kusstowa 16587.

Kutjanin 16666.

Kutscherowa 16539.

Kutschke 16426.

Kühnhanss 16707 .

Künne 16709 .

Kwate 16604

Kwlatek 16620.

Kymal 16894 .

Laakso 16667 .

Labbauf 16419 .

Labhart 16535.

Korschak 16665 .

Korver 16806.

16573. 16574 .

Labouesse 16513.

Ladell 16531.

Losmol 16524. Ladisch 16662 .

Kossol 16634. Ladyshenski 16633.

Kossolapowa 16402. Laffitte 16383 .

Kosszowa 16430. Laforet 16538 .

Kostyreff 16710. Lagunzow 16636.

Kotelnikow 16632. Lahiri 16607.

16492. Laikin 16367.

L'Air Liquide, Soc. An. pour l'stude et l'Exploitation dos Procédés G. 


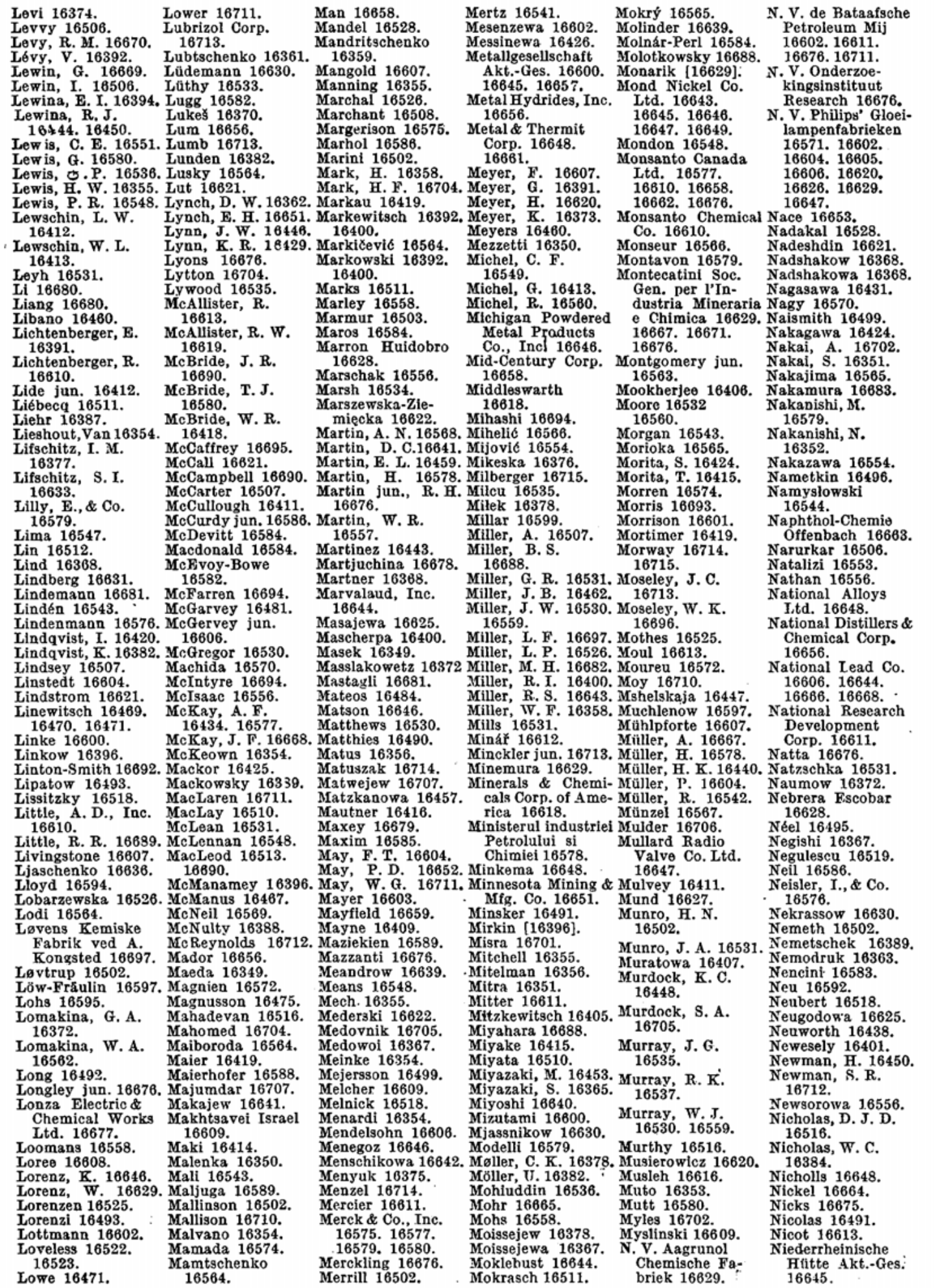


Niesler 16384 Nikitenko 16637 . Nikitin 16360 . Nikitine 16362 .

Nikolski 16378 .

Nilsson, G. Nilsson, K.T. 16659. Oth 16510 Nilson, N. R. 16696. Otsu 16603.

Nishii 16494.

Nishino 16579 .

Nishita 16549 .

Nisselsson 16897.

Niyogi 16593.

Noll 16679 .

Norddeutsche Affinerie 16626

Northover 16538.

Norton Grinding

Wheel Co. Ltd. 16649 .

Novak 16599 .

Novello 16575 .

Novotný 16612.

Nowikowa 16699.

Nowlin 16676.

Nowosselskaja 16582.

Nutting 16689.

Nuzzi 16709 .

Nyberg 16543 .

Obenshain 16699.

16352.

Overhoff, W.

Temper- und

Eisengießerei

16645.

Owen 16642.

Owtscharenko

16678.

Oyamada 16572 .

Pacewiczowa 16622. Petrow 16362.

Pachomowa 16588. Petrowa 16362.

Packer 16500 .

Padieu 16661.

Petrun 16408.

Pettit 16613.

Padkewitsch 16564. Petuchowa 16544.

Padmanabhan Pfau 16587.

$16390 . \quad$ Pfister 16578.

Page 16536. 16556. Pfitzer, C., \& Co.,

Paine 16686.

Pan 16393.

Paneth 16409.

Inc. 16580

16687 .

Pankeject 16605

Pannetier 16397. Phillips 16368.

Pantschenko 1638

Phillips Petroleum

16668. 16672 .

16427.

'Brien, J. I. 16674.

Ockerman 16380.

Odani 16375.

O'Donnell, I. J. 16701. 16527.

O'Donnell, J. M. 16625.

Oesterlein 16663.

Office National

d'Êtudes et de

Recherches Aéro-

nautiques,

O.N.F.R.A. 16647.

16250

Papojan [16565]

Paprocki 16356.

Parfenow 16532.

Paris 16434.

Parke, Davis \& Co. 16579.

Parker 16641.

Parrod 16492.

Parteni 16561.

Paschall 16684.

Pasini 16514.

Passowskaja 16608.

16676. 16712.

16713. 16715.

Phoenix-Rheinroh Akt.-Ges. Vereinigte Hüttenund Röhrenwerk 16646.

Piala 16561.

Pieroni 16547.

Pieters 16711.

Pilkuhn 16370.

Pilz 16523 .

Office National In- Passynski 16498.

Pinkalla 16697

Pino 16493. 16 16625 .

Ogg 16581. 16589.

Ogura, H. 16449 .

Ogura, Y. 16559.

Ohata 16559

Patel 16541. 16542. Pintér-Szakács

Paton 16484.

16584.

Patrick 16489. Piontelli 16604.

Patton, R. 16428. Piquett 16624.
Patton, S. 16691. Piragibe 16688.

Pauluzzi 16583.

Ohmura, T. 16360. Pavlík 16616.

Ohri 16621 .

Okami 16600.

Okamura 16494

Pisszow 16407.

Paweljewa 16394. Pittsburgh Plate
Pawlenko 16381.

Glass Co. 1661

Pawlowskaja 16498. Pitzer 16651.

Pixley 1635

Okun, L. B. 16350 .

Okun, M. G. 16669. Payne, M. G, 16623 .

Okssengendler 16461. Pearson 16618.

Plapp jun. 16553.

Plaskett 16533.

Oldham \& Son Ltd. Pechiney Comp. de Plate, Dr., G.m. 16606.

olds 16557.

Produits Chimiques et Électrométallurgiques

b. H. 16697

Olin 16402 . 16611. 16646.

mint Peirce 16508.

Olmstead 16662

Peissachowitsch

Olson, J. A. 16542. [16565].
Olson, R. L. 16689. Peiwe 16500.

Omura 15612 .

Onak 16477 .

Onaka 16862

Pelagalli 16542.

Penberthy 16612 .

Penn 16669.

O'Neal jun. 16710. Peperzak 16713.

Onoprijenko 16631. Perech 16697.

Opausszky 16356. Perekalin [16489].

Opel, A., Akt.-Ges. Perez-Mendez 16648 . 16350.

O'Rear 16654

Oreschko 16684

Perfogit S.p.A. 16662 .

Plate, N. A.

Platt 16568.

Platz 16662, 16672

Plechanow 16379 .

Plickat 16698 .

Pochwissnew 16631.

Pöhm 16437.

Pötner 16448.

Pogell 16510.

Pokraka 16444.

Pol 16633.

Polack 16712.

Poljakow 16388.

Pollock 16885 .

Polonsky 16487. 16489.

Orgins 16701.

Poluchin 16546.

tories Ltd. 16579. Perlmutter-Hayman Pomaska 16647.

Orlowa 16450. 16425.

Oroshnik 16661. Permachem Corp.

Orsino 16606. 16704.

Pond 16644 .

Pondel 16620.

Popják 16591.

Popovych 16361.

Porod 16489.

Porte 16416.

Posdejew 16639

Posin 16597.

Pospisil, R. 16640.

Pospišil, Z. 1661

Postma 16352.

tasse et Engrais Reimers 16570 .

Chimiques 16625. Rentsch 16430 .

Potter 16691.

Poutsiaka 16561.

Powell 16609.

Prasannan 16538

Pratesi 16579.

Preece [16687].

Price 16693

Pridanzew 16635 .

Pritchard, H. 0 .

16427.

Pritchard, R. 16507.

Prpié-Majić 16565.

Prochontschukow

16545.

Profft $1657 \%$

Proll 16377.

Prokopenko 16491. Ribrag 16359.

Prosser 16502. Richardson Co.

Prostoserdov 16686. 16606.

Protopescu 16371. Richards, H. L.

Pugatschew 16395. 16695.

Pugnet 16596 . Richards, R. E.

16415.

arvin \& Gertz, Inc. Richards, R. J.

16691 .

$\begin{array}{ll}\text { Puschhof } 16628 . & \text { Richter, K. } 16408 . \\ \text { Putschkowa } 16499 . & \text { Richter, R. H. H. }\end{array}$

Pyle 16355.

Pyrene Co. Ltd. 16649.

Pyzel 16619

Quayle 16513.

Quebec Iron \& Ti. tanium Corp. 16643.

\section{Raab 16665.}

abinowitsch

16641.

Raddatz 16572

adio Corp. of

America 16646 .

Radler 16685 .

16658 .

Radulescu 16522 .

Raha 16649.

Raible 16693 .

Ramb

Ramsden $\mathbf{1 6 6 6 1 .}$.

Ramseier 16535

Ramshorn 16526.

Randall 16555 .

Rank 16583. 16584. Rodiwilowa 16669

Rao, C. N. R. 16419. Rodot 16372 .

Rieck 16440.

Riehl 16557.

Rigan 16616 .

Rigden 16362 .

Ringwood 16405 .

Riniker 16477 .

Risol 16386.

Rivière 16441.

Robertson, A. V 16468.

Robertson, R. E. 16425.

Robertson 16614.

Robinson, A. E. 16521.

obinson.

Robinso

16527 .

obinson, J. W.

16582, 16709 .

Romo 16477.

Romo de Vivar

16484

omovacková

16708

Rosanove 16691

Rosanowa 16367.

Rosenberg, B. H. 16505.

Rosenberg, H.

16527.

Rosenbljum 16356 .

Rosenfeld 16597.

Rosenthal, A.J.

16704.

16677.

Rosenthal, W. A. 16697. 
Sandoz Soc. An. Schwarz 16548. 16572. Schwed 16635 . Sankyo Kabushiki Schweisheimer Kaisha 16572 . 16680.

Sansone jun. 16668. Schwenk 16598.

Sanyal 16706.

Sapovalov 16629.

Saric 16565 .

Sartorelli 16545.

Sastry 16516.

16646. $\mathrm{S}$.

Scott, K. P. 16618. 16463 .

Scott, W. E. 16579. Skinner, W. A.

Scoy, Van 16652 . 16443

Seaborg 16355. Skoog 16423

Satava 16612 .

Saunders jun. 16451. Sears 16556 .

Saussele jun. 16688. Sébille 16495.

Sauteron 16610. Seeburger 16657.

Saville 16663.

Sawicki 16593.

Sawyer 16528 .

Saxe 16709

Saxena 16351.

Saxty 16670.

Scatturin 16390.

Schabalin 16351

Schabarow 16444.

Schadijewa 16446 .

Schaefer 16415 .

Schaerf 16350 .

Seeley 16697.

Segrè 16351.

Seidel 16600.

Seifert 16599.

Seitz 16667 .

Seki 16629.

Sekiya 16561 .

Selbin 16403.

Seliger 16358.

Sellin 16540.

Senft 16531.

Senning 16440 .

Schalawina 16378. Serban 16486 .

Scharf 16583.

Scharff-Goldhaber 16354.

Schegk 16628.

Scheline 16551 .

Schenker 16477.

Schering Akt.-Ges. 16627.

Schering Corp. 16579

Sessner 16541.

Seth 16355 .

Seumel 16707.

Severini 16667 .

Sewin 18615.

Shabica 16575.

Shadan 16635.

Shak 16637.

Shakowa 16677

Shand 16613 .

Shankar 16390 .

Schiavo 16542 .

Schiefelbein 16599. Sharpe 16393.

Schiffler 16646. Shashoua 16415.

Schildknecht 16529. Shaw 16659.

Skoog 16423.

Skrishinskaja 16587

Skrotzki 16373.

Skrynnik 16669.

Skurichina 16497.

Skwartschenko 16450.

Skwortschuk 16

Slabecka 16624.

Slade 16428.

Slater 16503 .

Slaughter 16511.

Slee 16587

Slenczka 16504.

Slepko 16636.

Sloboda 16508.

Smetankina 16

Smeyers 16569.

Smialowski 163

16640 .

Smirnow, N. S.

16642.

Smirnowa, E. N. 16450 .

Smirnowa, J. P. 16669.

Smit 16425.

Smith, B. 16592. Spergely 16570

Smith, C. I. 16561. Spier 16647.

Smith, D. 16693. Spindler 16617.

Schilina 16381.

Schinz 16482.

Schirokschina 16402. Co. 16652 16660. Smith jun., H. R.

"Shell" Research 16644.

Ltd. 16628.

16713.

Smith, I. C. P.

Spiro 16509 .

Spitzyn 16398.

Spivey 16499. 16580 .

Spohn 16617.

Sheludew 16367, Smith, J. D. 16505. Spokes 16361.

Shibanuma 16472, Smith, J. M. 16599. Spray 16647.

Shibata, K. 16640. Smith, S. R. 16427. Spring 16484

Schkodinskaja 16442 .

Schlicke [16606].

Shibata, T. 16493

Shigeno 16684 .

Shimamoto 16562

Shimamura 16512 .

Shiobara 16574

Shipton 16690 .

Shomate 16418 .

Shorley 16536.

Thidt, B. J. M. 16619 .

moczkiewiczowa Sprung 16496.

$$
16543 .
$$

Smrt 16660 .

Snart 16498.

Snegur 16637.

Snow 16358.

Schmidt, E, 16518. Shri Ram Institute Sobel 16548.

Schmidt, Ernst [16595].

Schmidt, K.-H. 16664 .

Schmidt, P. 16660. Shute 16548.

Schmitt 16573 .

Schnegg 16697.

Schneider, A. 16648. Siegrist 16663.

Schneider, G. 16371. Siemens-Plania-

Schönberg 16449 .

Scholl 16684.

Scholz, M. 16697.

Scholz, R. 16448 .

Schosstakowski 16665.

Schrader 16628.

$$
16629 .
$$

Schreider 16643

Schueler [16580].

Schunemann 16458 .

Schuldiner 16380.

Schulek 16584.

3chuler 16577.

Schultze 16714 .

Schulze 16616.

3chur 16374.

Schwanda 16683.

3chwartz 16434 .

3chwartzberg

16638.

ques d'Ugine
16661. for Industrial

Shugar 16513.

Shukow 16635 .

Sieg 16651.

Siegenthaler

werke Akt.-Ges. für Kohlefabr kate 16649 .

Siemens-Schuckertwerke Akt.-Ges. 16619.

Sieskind 16362.

Silva 16354.

Silver 16388.

Simakow 16359.

Simonds [16677].

Sinclair 16555.

Singer, J. J. 16656.

Singer, S. J. 16529.

Singh, Sarabjit 16619.

Sobotka, M.

. 16581. Ssawina 16544 .

Sobotka, Z. 16612. Sseljawo 16640 .

Alan- Sserebrjakowa chiments, Teintu- 16642 res \& Impressions Ssinani 16372 . 16703 .

Ssinitzki 16632 .

c. An. des Etablis- Ssokolowa 16397. sements Parra- Ssologub 16594. Mantois \& Cie. Ssolowjewa 16464. 16620.

Ssolowjewa 16642 .

oc. An. des Manu- Ssuchanowski factures des Gla- 16669.

ces et Produits Ssudakow, P. J.

Chimiques de

Saint-Gobain

Chauny \& Cirey

Soc. An. Turbinia 16650

Soc. d'î́lectro-Che-

16708.

Ssudakow, W. W. 16350.

Ssuikowskaja 16402. Subenko 16472

dectro- - Ssurin. 16637.

, des Ssurowow 16637.

Aciéries Electri- Ssuschko 16430.

Ssuschko 16430 .

Stammler 16530 .

Gru- Suda 16515.

Sinha 16706.

Sirtl 16400 .

Sistrunk 16689 ments Stobel

ber \& Co. 16679. Süling 16675 . 16081.

Stamboulian 16700 .

Soc. For 16649 .

Standard Oil Co. Sunagawa 16572 Sun Oil Co. 16711

Standard Oil Deve- Sutaria 16492.

Sutcliffe 16377.

Sutherland 16655 .

Stanley 16593.
Starodubow 16392 . Suzuki 16417.

Starschinow 16632. Svehla 16587.

Svennerholm 16540

Svenska Ackumulator Aktiebolaget Jungner 16605.

Svenska Mjollkprodukter A/B

16696.

Swabb jun. 16711.

Sweetman 16366 .

Swern 16655.

Swirski 16361.

Swoboda 16375 .

Sykes 16551.

Sylvania Electric Products, Inc. 16605. 16606.

Szab6 16608.

Szarvas 16585 .

Szczypiński 16624 .

Szeinberg 16511.

Sztumpe 16513 .

Tabata 16353.

Taborsky 16569 .

Tada 16535 .

Tănzler 16701.

Täufel 16500 .

Tager 16369 .

Takagi 16559

Steyermark 16590. Takahuwa 16643.

16590 .

Stine 16715.

Takaki 16415.

Takaori 16557 . 16559.

Stock 16522. 16523. Takayama 16365.

16713.

cheff 16412.

Stoll, A. 16576. 
Thomas, W. N. 16493.

Thompson, B. R. 16674.

Thompson, E. O. 16527. 16701 16703.

Thompson, J. D. 16398.

Thompson, S. G. 16355 .

Thomsen 16570 .

Thormann 16406 .

Thuan 16494

Tíchomirowa 1663

Tiedeman 16654 .

Tighe 16551.

Timmins 16631.

Timmler 16518.

Tinker 16473 .

Tinoco jun. 16

Titan Co., Inc. 16668.

Titarenko 16582.

Tjutjunnikowa 16366.

Tkalitsch 16564.

Tkatschenko

Toby 16418 .

Tölgyessy 16582 .

Toh 16536.

Tokarski 16625.

Tokita 16560

Tokuyama Soda $\mathrm{K}$ abushiki $\mathbf{K}$ aisha 16600 . Toltschinski $16496 . \quad 16560$.

Tomasini 16354.

Tomassi 16378 .

Tomko 16565.

Toole 16375 .

Toone 16666

Toptschijew 16496.

Torgeson 16521.

Torres 16669 .

Towle 16713.

Toyoda 16349 .

Toyomoto 16678.

Trabanelli 16591.

Transcontinental

Development Co.

Trust Reg 16697. U

Trauffer 16614.

Travis 16557

Treschtschowa

16444. 16450.

Tribolet, de 16482 .

Troelstra 16604.

Troester 16605.

Trojanowski 1

Tromp 16356

Tronchet, A. 16526 .

Tronchet, J. 16526.

Tron-Loisel 16560.

Trotman16423.

Trott 16358.

Troxell 16615 .

Truchot 16560 .

Trüb 16595.

Truett 16676.

Truscheit 16576.

Truswell 16643.

Tschajewski 16634.

Tschalenko 16686.

Tschassowitin 16632.

16666.

16662 . 16417. 16454. 16697. 16667. 16643. 16662. 16654. 16711.

16667.
Tschekin 16630 .

Tschertschess

16669. Usui 16561.

16356. Utley 16614

Ts'o 16505 .

Vagi 16641.

uneto 16373.

unoda 16579

Tuba 16437.

Tuckey $\mathbf{1 6 5 4 9}$

Tumanow 16702 .

Tunin 16569.

Turbelier 16602.

Tuthill 16594 .

Tye 16605.

Tyree 16599.

Ubbelohde 16396 .

ULAF 16682

Uchiyama 16629

Udupa, H. V. K.

Udy 16688 .

Udylite Research Corp. 16605.

Újhidy 16708

Ukita 16431. 16453 .

Ulfstädt 16619 .

Union Carbide \& Carbon Corp. 16605. 16606.

Union Carbide Co 16610. 16620 . 16672. 16674 .

Union Chimique Belge Soc. An.

United Kingdom Atomic Energy

nited States of

America, Secretary of Agriculture 16655. 1665

United States of America, Secre tary of Army

United States of America, Secretary of Navy

United States Ato mic Energy Com-

United States Corp. 16611.

United States Steel Walker, S. 16615 Corp. 16668.

Universal Oil Products Co. 16651.

Unna 16557.

Unterstenhöfer

Vaisler 16535.

16375.
Updegraff 16608.

Urnes 16612 .

Vacea 16542.

Vagramjan 16378.

aíro 16519.

Valensin 16681 .

Valk 16350.

allee 16547 .

Valmont 16528.

Vandenberg 16676 . Vardheim 16698 .

Varner 16519.

Varvill 16668.

Vasiliu 16358

Vaugham 16616 .

Vaz 16519.

VEB Filmfabrik

16611.

Watkins 16711 .

Venugopalan 16538. Wayman 1665

Verga 16539.

Verters 16610.

Vesterdal 16714

Viallet 16401 .

Vigh 16586.

Vilcsek 16409.

Vingas 16630.

Vinograd 16537.

Visconti 1661

Vitrone Jun.

Vogt 16531.

Voigt, G. 16606.

Voigt, J. L. 166

Volkin 16505 .

Volpi 16366.

Vona 16661 .

Voss 16533.

Wachtel 16604.

Waddell 16546.

Wänke 16409 .

16410.

Wagner, A. 16700 .

Wagner, G. 166 16582 .

Wahlig 16529.

Wainstein 16635 .

Waite 16662

Walenta 16407.

Walery\$ 16414.

Walijew 16377 .

Walker, G, C.

16516.

Walker, G. P. I.

J. 16554.

16558.

Walker, $T$. $\mathbf{K}$.

16519.

Wallenfels 16512 .

Wallquist 16602 .

Walser 16533.

Walsh, A. 16360.

Walsh, D. E. 16375 . T. A.

Wang 16520 .

Warner 16656.

16563 .

Wassima 16442 .

atanabe, $\mathbf{H}$.

16453. 16454. 16455 .

Watanabe, M. 16496.

Watari 16352

Watelle-Marion

16404.

Watt 16379 .

Wayman 16653 .

Weaver 16606 .

Webber 16687 .

Webster 16587 .

Wedler 16385 .

Weí 16670 .

Weidmann 16475 . 16476.

Weil 16644.

Weiland 16651.

Weimer 16518 .

Weis 16529 .

Weisberg 16656.

Weiss 16700 .

Weiszburg 16364

Weitz 16672.

Wellcome Foundation Itd. 16572.

Welsh 16432.

Wender 16708.

Wendler 16614

Wenzel 16552 .

Wereschtschagin 16395.

Valsh, J, J, 16537. Wildman 16594.

Valsh, T. J 16589. Wilenzick 16355 .

Willard 16353

, Williams, C. T.

W. 16541. 16690 .

Williams, L, A.

16473.

. Williams, R. T.

16551.

Williams, R. W.

16349. 16660.

mson, K. S. 16531.

Willis, C. J. 16429

Willis, J. B. 16595

Wilson, H. 16556.

Windgassen 16477. Wyckoff 16477 .

Winding [16677].

Winer 16561.

ingfoot Corp. 16676 .

Winkel 16604.

nogradow, $\mathbf{L}$

16614

Winogra

16365 .

Winter, W. 16596. Ges. 16626.

Wintle 16490. 
Patentregister

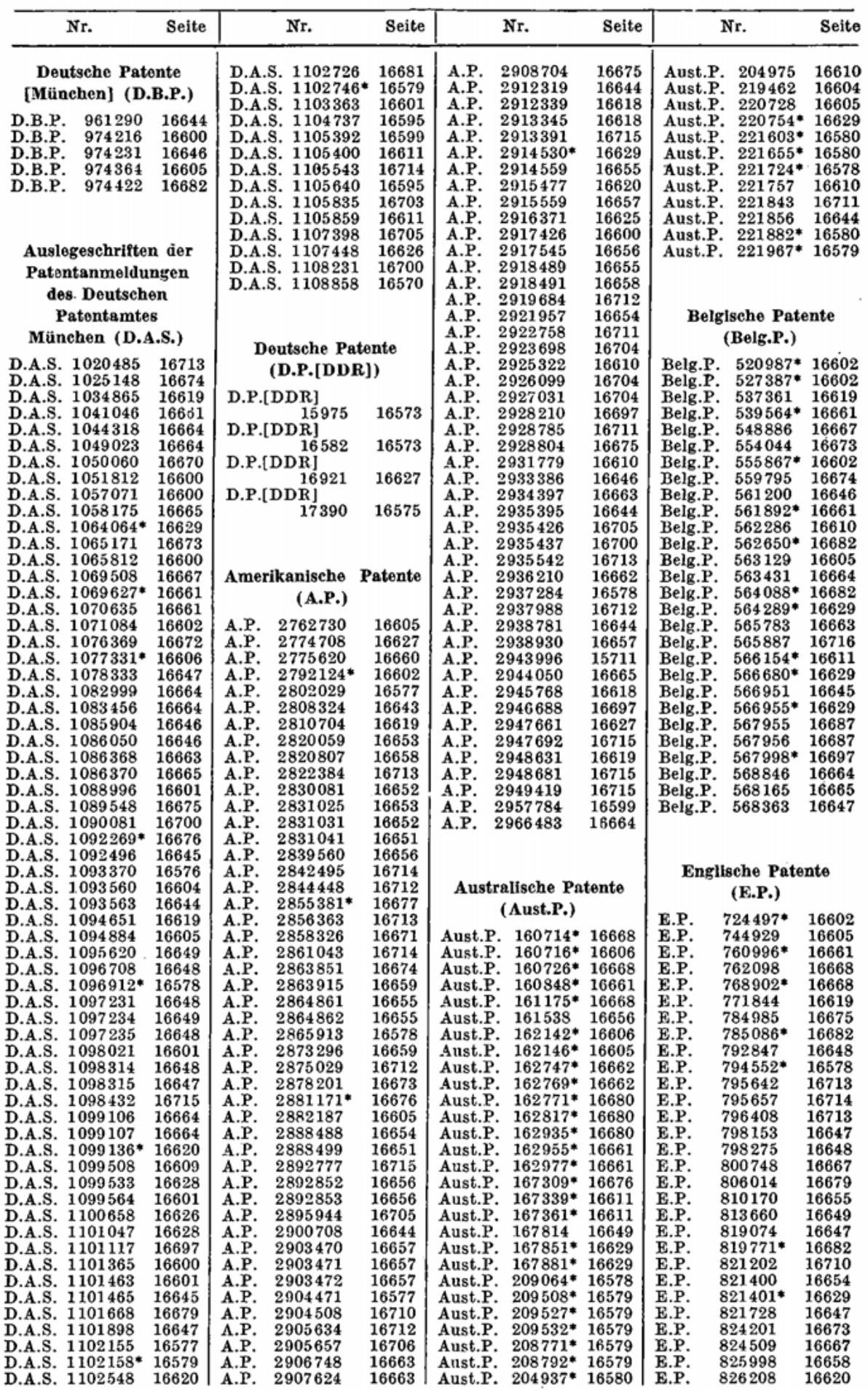




\begin{tabular}{|c|c|c|c|c|c|c|c|c|c|c|c|}
\hline & Nr. & Seite & & Nr. & Seite & & Nr. & Seite & & Nr. & Seite \\
\hline & & & & & & & 1197583 & & & & \\
\hline & & & & $1149031^{*}$ & 16606 & F.P. & & 16628 & Ind.P. & $56451^{*}$ & 16668 \\
\hline & 830738 & 16609 & F.P. & $1149462^{\circ}$ & 16606 & F.P. & 1198268 & 16572 & Ind.P. & $57033^{*}$ & 16580 \\
\hline & 831420 & & F.P. & 1150709 & 16716 & F.P. & & 16573 & Ind.P. & $57047^{*}$ & 16580 \\
\hline & 831745 & 16610 & F.P. & $1151365^{*}$ & 16676 & F.P. & 1198607 & 16576 & Ind.P. & $57110^{*}$ & 16602 \\
\hline E.P & 833982 & 16610 & F.P. & $1151366^{*}$ & 16676 & F.P. & 1198741 & 16660 & Ind.P. & $57116^{*}$ & 16697 \\
\hline & & 16611 & F.P. & $1151759^{*}$ & 10668 & F.P. & 1198889 & 16657 & Ind.P. & $57142^{*}$ & 16579 \\
\hline & $834599^{*}$ & 6662 & F.P. & $1152145^{*}$ & 16668 & F.P. & 1199324 & 16650 & Ind.P. & $57200^{*}$ & 16611 \\
\hline E.I & $834883^{*}$ & 16676 & F.P. & $1153101^{*}$ & 16676 & F.P. & 1199352 & 16658 & Ind.P. & $57269^{*}$ & 16602 \\
\hline & $835385^{*}$ & 16682 & F.P. & $1153312^{*}$ & 16676 & F.P. & 1202064 & 16600 & Ind.P. & 57353 & 16679 \\
\hline & 835927 & 6704 & F.P. & $1153313^{*}$ & 16677 & F.P. & 1205373 & 16664 & Ind.P. & $57461^{\circ}$ & 16579 \\
\hline E.I & 836098 & 16643 & F.P. & 1153457 & 16668 & F.P. & 1205881 & 16663 & Ind.P. & $57462^{*}$ & 16579 \\
\hline & 836340 & 16705 & F.P. & $1154331^{*}$ & 16679 & F.P. & 1207331 & 16648 & Ind.P. & $57468^{*}$ & 16579 \\
\hline & $836896^{*}$ & 16661 & F.P. & $1154452^{*}$ & 16676 & F.P. & 1211508 & 16664 & Ind.P. & $57478^{*}$ & 16580 \\
\hline E. & $836897^{*}$ & 16661 & F.P. & 1157845 & 16671 & F.P. & 1212336 & 16648 & Ind.P. & $57483^{*}$ & 16649 \\
\hline & 836980 & 16611 & F.P. & $1159805^{*}$ & 16605 & F.P. & 1213033 & 16704 & Ind.P. & 575 & 16646 \\
\hline E. & 8372 & 16600 & F.P. & $1160720^{*}$ & 16676 & F.P. & 1213292 & 16646 & Ind.P. & 57603 & 16700 \\
\hline & 838 & 16600 & F.P. & $1161078^{*}$ & 16676 & E.P. & 1213450 & 16645 & Ind.P. & $57676^{*}$ & 16580 \\
\hline & $838752 *$ & 16676 & F.P. & $1161238^{*}$ & 16679 & F.P. & 1214194 & 16647 & Ind.P. & & 16673 \\
\hline E. & 8391 & 16711 & F.P. & 116 & 16671 & F.P. & 1215108 & & Ind.P. & $57761^{*}$ & 16579 \\
\hline & 8401 & 16629 & F.P. & $1166558^{*}$ & 16679 & F.P. & 1215658 & 16605 & Ind.P. & $57777^{*}$ & 16578 \\
\hline E. & 840 & 16611 & F.P. & $1166559^{*}$ & 16679 & F.P. & & & & & 16579 \\
\hline E. & 8403 & 16649 & F.P. & $1167449^{*}$ & 16661 & F.P. & 1215891 & & Ind. $P$. & & 16601 \\
\hline & $840379^{*}$ & 16611 & F.P. & $1168027^{*}$ & 16676 & F.P. & 1216501 & 16681 & Ind.P. & 578 & 16643 \\
\hline E. & 841413 & 16664 & F.P. & 11 & 16679 & F.P. & & & I.P. & & 16619 \\
\hline E. & 8417 & 16646 & F.P. & 116 & 16651 & F.P. & & & & & 16578 \\
\hline & $841819^{\circ}$ & 16697 & F.P. & 1173200 & 16673 & F.P. & 1217549 & 16682 & Ind.P. & 579 & 16672 \\
\hline E. & 841 & 16662 & F.P. & 11 & 16705 & F.P. & & & & & 16579 \\
\hline E. & 8431 & 16704 & F.P. & 117 & & F.P. & & & & & \\
\hline & & 16644 & F.P. & 1182058 & 16625 & F.P. & 1219548 & 16700 & Ind.P. & $37 *$ & 16606 \\
\hline E. & & 16600 & F.P. & 11 & 16673 & F.P. & & & & & 16672 \\
\hline E. & 845 & 16645 & F.P. & 118 & 16611 & F.P. & & & & & \\
\hline E. & & 16627 & F.P. & 1182882 & 16609 & F.P. & 1222495 & 16645 & Ind.P. & 58563 & 16673 \\
\hline & & 16605 & F.P. & 118 & 16626 & F.P. & 122 & & & & 16645 \\
\hline & 8465 & 16687 & F.P. & 118 & 16661 & F.P. & 48 & 166 & & 8* & 165 \\
\hline E.I & & 16696 & F.P. & 118 & 16610 & F.P. & 1228461 & 16644 & Ind.P. & $58693^{*}$ & 16580 \\
\hline & & 16665 & F.P. & 118 & 16574 & F.P. & $1229649^{*}$ & 16611 & Ind.P. & & 16620 \\
\hline & 847728 & 16712 & F.P. & 118 & 16573 & & & & Ind.P. & 587 & 16579 \\
\hline E. & 8479 & 16605 & F.P. & 118 & 16659 & & & & Ind.P. & $58733^{*}$ & 16579 \\
\hline & & 16687 & F.P. & & & \multirow{4}{*}{\multicolumn{3}{|c|}{$\begin{array}{c}\text { Holländische Patente } \\
\text { (Holl.P.) }\end{array}$}} & & & 16 \\
\hline & 849 & 16599 & F.P. & 118 & 16572 & & & & Ind.P. & $58795^{*}$ & 16579 \\
\hline E.I & $849585^{*}$ & 16697 & F.P. & 1187762 & 16650 & & & & Ind. & 58817 & 16656 \\
\hline & & 16664 & F.P. & 1187799 & 16660 & & & & & & 16620 \\
\hline E.F & $850176^{*}$ & 16661 & F.P. & 1187890 & 16656 & Holl.P. & 92505 & 16674 & Ind.P. & $58831^{*}$ & 16580 \\
\hline E. & & 16664 & F.P. & 118 & 16651 & Holl.P. & & & & & 16658 \\
\hline & & 16687 & F.P. & & & Holl.P. & $95431^{*}$ & & & & \\
\hline & 850655 & 16618 & F.P. & 118 & 16660 & Holl.P. & $95444^{*}$ & 16 & Ind.P. & & 16619 \\
\hline & & 16711 & F.P. & 118 & 165 & Holl.P. & $95449^{*}$ & 165 & & & 16580 \\
\hline & & & F.P. & & & Holl.P. & 954 & & & & \\
\hline E. & $851690^{\circ}$ & 16611 & F.P. & 118 & 16664 & Holl.P. & $95464^{*}$ & 16629 & Ind.P. & & 16620 \\
\hline E. & & 16676 & F.P. & & & Hol & 9553 & & & & 16602 \\
\hline & & & F.P. & & & Holl.P. & & & & & \\
\hline E.1 & $852180^{*}$ & 16611 & F.P. & 118 & 16651 & Holl.P. & 9575 & 16676 & Ind.P. & 59 & 16 \\
\hline & & & F.P. & & & Hol & & & & & \\
\hline & & & F.P. & & & Holl.P. & & & & & \\
\hline E. & 856430 & 16605 & F.P. & 119 & 16662 & Holl.P. & $95909^{*}$ & 16662 & Ind.P. & $59517^{*}$ & 16579 \\
\hline E.1 & & 16664 & F.P. & 119 & 16652 & & & & Ind.P. & 599 & 16652 \\
\hline E.F & & 16705 & & & & & & & Ind.P. & & 16609 \\
\hline & & & & & 16661 & & & & Ind.P. & 600 & 16579 \\
\hline & & & & & 16660 & Indi & ische Pate & nte & P. & & 16580 \\
\hline & & & & & 16659 & & (Tnd & & Ind.P. & $60313^{*}$ & 16578 \\
\hline & sische & ente & & $1190615^{*}$ & 16662 & & & & Ind.P. & 60394 & 16628 \\
\hline & & & & & 16652 & Ind.P. & $49140^{*}$ & & & & \\
\hline & & & & & & & & & & & \\
\hline & & & $\begin{array}{l}\text { F.P. } \\
\text { F.P. }\end{array}$ & & $\begin{array}{l}16661 \\
16651\end{array}$ & $\begin{array}{l}\text { Ind.P. } \\
\text { Ind.P. }\end{array}$ & $\begin{array}{l}49192^{*} \\
49199^{*}\end{array}$ & & Italle & nische $\mathrm{Pa}$ & \\
\hline & $187^{*}$ & 166 & F.P. & & 16674 & Ind.P. & $49265^{*}$ & 16668 & & (It.P.) & \\
\hline & & 167 & F.P. & & & Ind.P. & 49285 & 16611 & & & \\
\hline & & & & & & & & & & & \\
\hline & & & & & & Ind.P. & 493 & & & & \\
\hline & & & & & & & & & & & \\
\hline & & & & & & & * & & & & \\
\hline & & & $\mathrm{F}$. & & & Ind.P. & & & & & \\
\hline & & & & & & & & & & & \\
\hline & 112 & & & & & & 49 & & & & \\
\hline & & & F. & & & & & & & & \\
\hline & & & & & & & & & & & \\
\hline & & & & & & & 49 & & & & \\
\hline & & & F. & & & & & & & & \\
\hline & & & F. & & & Ind.I & & & It. & & \\
\hline & 114350 & & & & & Ind.P. & 499 & & & & \\
\hline & & & F.P. & & & Ind.P. & & & It.P. & & \\
\hline & & & & & & & 56 & & It. & & \\
\hline F.P. & $1148046^{*}$ & 16605 & F.P. & 1197582 & 16573 & Ind.P. & $56323^{*}$ & 16668 & It.P. & $514607^{*}$ & 16580 \\
\hline
\end{tabular}




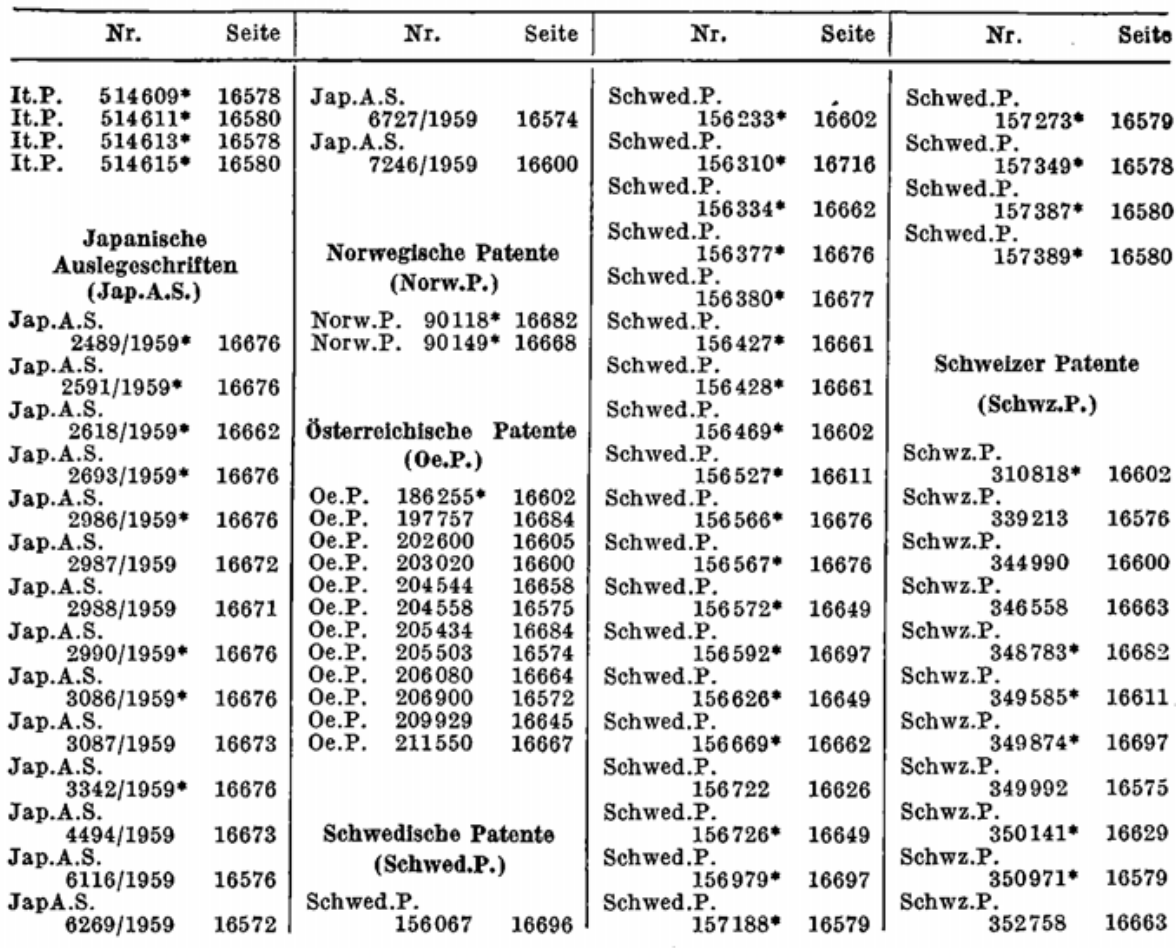

Der Fachkatalog

\section{CHEMIE}

gibt allen Interessenten einen umfassenden Uberblick über unsere einschlägige Literatur.

Er informiert über Inhalt, Umfang, Ausstattung und Preise der Veröffentlichungen der

\section{Deutschen Akademie der Wissenschaften zu Berlin, Sächsischen Akademie der Wissenschaften zu Leipzig, deren Institute und Forschungsstätten.}

Angaben über die BEZUGSMÖ GLICHKEITEN im In- und Auslande sind gleichzeitig enthalten.

Kostenlose Lieferung auf Anforderung beim Verlag.

Für andere Wissensgebiete stehen ebenfalls Fachkataloge und Einzelprospekte zur Verfügung. Interessenten erhalten bei Bekanntgabe von Anschrift und Fachgebiet unverbindlichSpezialangebot ihrer Fachliteratur und laufend Informationen über Neuerscheinungen.

A K A D E M I E •VE RLA G • B E R LI N 


\section{Chemie und Technologie der Silicone}

Unter Mitarbeit von

Dr. Oskar Glenz, Leverkusen; Prof. Dr. Gerhard Hecht, Wuppertal-Elberfeld; Dr. Walter Krauss, Leverkusen; Dr. Julius Peter, Leverkusen; Prof. Dr.-Ing. Hubert Rothert, Berlin; Dr. Bruno Zorn, Leverkusen

$1960 \cdot \mathrm{XV}, 460$ Seiten mit 101 Abbildungen und 97 Tabellen sowie etwa 2000 Literaturzitaten - Ganzleinen DM 39,80

Aus Besprechungen:

„... Das vorliegende Buch von Prof. Dr. Walter NOLL über ,Chemie und Technologie der Silicone' nimmt eine Sonderstellung ein, weil es das gesamte Silicongebiet von den Monomeren über die Polymere bis zu den technischen Produkten behandelt. Für die Anwendung der technischen Siliconprodukte in den verschiedenen Industriezweigen hat der Verfasser jeweils Experten zur Mitarbeit herangezogen..."“

TEXTIL PRAXIS

„... Den Verfassern ist es in hervorragender Weise gelungen, das Gebiet der Chemie und der Technologie der Silicone zusammenzufassen. Die zahlreichen Tabellen und graphischen Darstellungen, die Vielzahl der Literatur- und Patentzitate, sowohl für den chemischen wie für den technischen Teil einerseits und die kritische Bearbeitung, die souveräne Beherrschung und die geschickte Darstellung des Steffes andererseits, machen das Buch für den Hochschullehrer und Studierenden, für den Chemiker, Physiker und Mediziner, für den Techniker und den Anwendungsfachmann zu einem Standard-Werk, welches sich auch in nichtdeutschsprachigen Ländern seinen Platz sichern wird.“

KUNSTSTOFFE

Prospekt auf Wunsch

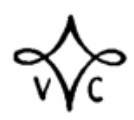

VERLAG CHEMIE - GMBH - WEINHEIM / BERGSTR. 